\title{
Agentes educativos y multiculturalidad en el aula
}

\author{
Antonio Rodríguez Fuentes ${ }^{1}$ \\ Alejandro Daniel Fernández Fernández²
}

\section{Resumen}

La profusión de escolarización de alumnado inmigrante y de otras culturas junto a la proliferación de patrones escolares inadecuados de acogida y episodios lamentables en torno a su tratamiento, hace preciso el estudio y optimización actitudinal de profesores, alumnos y padres/madres. Solo la concordancia/coherencia perceptivo-actitudinal en torno a patrones adecuados por parte de ellos evitará el caldo de cultivo de conflictos mal resueltos responsables de la reproducción de episodios no deseados. Se entrevistó a tres sectores o casos de distintas características y contextos para garantizar la representatividad, de cara al establecimiento de patrones y diferencias entre colectivos así como las de dentro de ellos. Para ello, se han categorizado, y debidamente codificado, las declaraciones de cada entrevistado, mediante un sistema de categorias a posteriori, al carecer de modelo teórico consolidado del fenómeno que propiciara el empleo de categorías apriorísticas. Posteriormente, se ha practicado el consecuente análisis de contenido por agrupación y reducción de categorías a través de metacategorías; de tal arte que se han detectado patrones perceptivos no siempre adecuados en los colectivos estudiados, en torno a la información sobre otras culturas y formación para su tratamiento. Se han sometido los datos a análisis porcentuales y diferenciales que apuntan divergencias significativas entre progenitores y profesores, así como entre ellos y los hijos o alumnos. Menores son las diferencias internas entre miembros de cada sector. Siendo las primeras mayores que las segundas, como es el caso, existe un conflicto generacional que requiere ser resuelto.

\section{Palabras-clave}

Actitudes ante la diversidad cultural - Percepciones ante la diversidad cultural - Agentes educativos.

\footnotetext{
1- Universidad de Granada, Granada, España. Contacto: arfuente@ugr.es

2- Consejería de Educación y Ciencia, Granada, España. Contacto: alejandro_dfernandez@hotmail.com
} 


\section{Educational agents and multiculturalism in the classroom}

\section{Abstract}

The dissemination of schooling of immigrant students and from other cultures together with the spread of inadequate school patterns of welcome and regrettable events concerning the ways these students are treated makes it necessary to study and to optmize the attitudes of teachers, students, and parents. Only the perceptive-attitudinal agreement/ coherence around suitable patterns will prevent the cultivation of conflicts badly resolved that lead to the reproduction of unwanted situations. Three sectors or cases of different characteristics and contexts were interviewed in order to ensure representativeness, with the purpose of establishing patterns and differences between the groups and among them. For such, the statements of each interviewee were categorized e duly coded by using a system of a posteriori categories, in the absence of consolidated theoretical framework regarding the phenomenon which would have allowed the use of a priori categories. Further, content analysis was carried out by grouping and reducing the categories by means of metacategories; thus, perceptive patterns, not always appropriate, were detected in the groups studied, around the information of other cultures and formation for its treatment. Data have undergone percentage and differential analyses that point to significant divergences between parents and teachers as well as between them and their children and students. Minor are the internal differences among the members of each sector. Since the first ones are bigger than the second ones, as it appeared in this study, there is a generation conflict that needs to be resolved.

\section{Keywords}

Attitudes towards cultural diversity - Percepctions towards cultural diversity - Educational agents.

\section{Introducción}

La diversidad cultural en la sociedad y centros educativos es una realidad incuestionable y se hace evidente para la gran mayoría de las personas por los episodios dramáticos que con frecuencia se producen en las relaciones interculturales. Conductas que son producto de actitudes y percepciones erróneas hacia la alteridad, hacia otras culturas, creencias, hábitos y a veces simplemente opiniones discrepantes. De ahí surge la justificación de esta investigación por su pretensión de dilucidar los patrones perceptivoactitudinales de los agentes educativos más importantes: profesorado y alumnado, como representantes directos del acto didáctico, y padres/madres del alumnado en tanto que referentes. Es, en principio, un objetivo descriptivo por cuanto pretende resaltar las 
declaraciones más frecuentes de cada colectivo, tomándolas como patrón comportamental de cada uno, agrupándolas según concordancia entre ellos. Pero tan importantes se conciben las coincidencias como las diferencias entre grupos, además de intragrupos, para ver qué colectivos están más cercanos en cuanto a sus patrones declarados, y en su caso, quiénes influyen más en la configuración del alumnado: progenitores o profesores. Complementa al objetivo descriptivo anterior éste de tipo inferencial.

Existe cierta tradición en la indagación de las cualidades perceptivo-actitudinales profesorales ante la diversidad cultural, sus expectativas, su formación inicial y permanente, etc. en contextos de interculturalidad. Los estudios enfatizan las carencias y deficiencias mostradas por el colectivo del profesorado, consecuencia, en parte, del tránsito de la antigua realidad monocultural de sus aulas a la evidencia multicultural contemporánea, y por tanto sus nuevas demandas y disfunciones para la atención al alumnado de culturas minoritarias (ETXEBARRIA; INTXASUTI, 2013; ETXEBARRIA; KARRERA; MURUA, 2010; GÓMEZ, 2015; LEIVA, 2012; MERINO; LEIVA, 2007; MERINO; RUIZ, 2005; MOLINER; MOLINER, 2010; SALAS et al., 2012).

Algunas investigaciones, en menor cantidad, se han realizado tomando como fuente de datos el alumnado inmigrante o de culturas minoritarias, dando esa voz reclamada por distintos enfoques de investigación a los directamente afectados por las deficiencias del sistema y de sus agentes (CALVO, 2008; SALAS et al., 2012 RUIZ; MERINO, 2009). No es el caso de la presente investigación, pues en esta ocasión se orienta hacia los alumnos pertenecientes a la cultura mayoritaria, y a su preparación actitudinal y perceptiva para convivir en escenarios multiculturales, máxime cuando las investigaciones en este sentido son muy escasas (BARRENA, 2014).

Igual acontece con las investigaciones familiares realizadas sobre culturas minoritarias (ETXEBARRIA; INTXAUSTI; JOARISTI, 2013; LEIVA; ESCARBAJAL, 2011; SALAS et al., 2012; SANTANA; FELICIANO; JIMÉNEZ, 2016; SANTOS; LORENZO, 2009; SANTOS; LORENZO; PRIEGUE, 2011). En esta ocasión se han entrevistado a los de la cultura mayoritaria, cuyos hijos están escolarizados en centros en los que existe gran diversidad cultural, por tanto están afectados de la misma.

Algunos autores incluso han combinado algunos de los tópicos anteriores, como se ha realizado en esta ocasión, pero siempre tomando como fuente los alumnos y familias de culturas minoritarias, al contrario de lo que se ha contemplado en la presente investigación. Son casos testimoniales, como las investigaciones desarrolladas por Intxausti, Etxebarria y Joaristi (2014) que estudian docentes y familiares; por Merino (2005), sobre percepciones profesorales y actitudes discentes; o por Aguado y otros autores (2003), cuyo foco es el de los tres colectivos: profesores, progenitores y alumnos. Y este último ha sido precisamente el foco de la tesis doctoral de la cual se presentan los datos en este momento, siempre desde la perspectiva de las percepciones y actitudes de los colectivos anteriores pertenecientes a la cultura mayoritaria, y no a la minoritaria, como los estudios existentes.

Se define actitud como la postura, disposición o determinación del ser vivo ante una situación o fenómeno, que predispone un estado de ánimo e incluso, en gran medida, una ventana hacia la acción. Ésta está influida y a su vez repercute en el plano teórico en la percepción, entendida como el conocimiento e idea de la situación o fenómeno. Sin embargo, existen determinantes complementarios de las anteriores y responsables a su vez 
de la conducta mensurable final. Se trata de la dimensión social y, muy particularmente, en el caso de menores, cuyo grado de maleabilidad es mayor, de la dimensión familiar y escolar, a partes no bien identificadas.

La adaptación de individuos de culturas minoritarias, diferente a la mayoritaria, no depende exclusivamente de ellos y sus familias, sino que responde a la acogida mostrada por parte de otros miembros de la cultura mayoritaria. En el caso de los menores, concretamente el alumnado, depende de la cultura escolar y el clima del aula, fuertemente determinados por sus agentes educativos básicos, profesorado y alumnado, así como los progenitores en tanto que referente actitudinal de ellos, principalmente del alumnado.

Dada la profusión de la escolarización de alumnado inmigrante y de otras culturas minoritarias, que hace de la realidad escolar un contexto multicultural contrario a la obsoleta tradicional monoculturalidad, junto a la proliferación de patrones inadecuados de acogida dentro de las aulas y centros, en ocasiones de envergadura y calado alarmantes, se justifica la investigación en torno al estudio perceptivo-actitudinal de los agentes educativos fundamentales: profesores, alumnos y familias. Pero se añade, en esta ocasión, la concordancia, correlación y discrepancia entre unos colectivos y otros, lo cual informa: a) de una parte, sobre la influencia, en su caso, entre ellos, evidentemente de mayores (profesorado y familiares) a menores (hijos-alumnos); b) de otra parte, sobre la potencial confusión actitudinal-perceptiva que pudiera generarse en los menores, como consecuencia de la desavenencia, en su caso, de los mayores.

\section{Método}

La investigación desarrollada responde a un diseño transversal de tipo no experimental por estudio de casos, que combina la metodología cualitativa con la cuantitativa. En efecto, se estudian tres casos, que coinciden con los tres colectivos arriba aludidos de distintos centros de la provincia de Granada, cursos, zonas, tamaños y poblaciones inmigrantes como garante de representatividad cualitativa. Por supuesto, las características intrínsecas de los participantes también son diferentes (edad, sexo, antigüedad, nivel de estudios, etc.). Aunque los sujetos han sido elegidos intencionadamente (muestra de conveniencia) como permite el estudio de casos, se ha tenido en cuenta la representatividad de la muestra ${ }^{3}$, más bien sujetos de la investigación, con respecto a la población a la que responden. Dentro del colectivo de profesorado que imparte en los centros y niveles reseñados, la población es de 36, por tanto, la muestra ha de ser de 24, como así es. La población de padres conocedores del centro y su diversidad es, según declaraciones de tutores de esos niveles y centros, de 32 , de ahí que se requiera una muestra de 22, siendo tales los padres y madres entrevistados. Por último, del total de 360 alumnos que reúnen los requisitos anteriores se precisa una muestra de 58, siendo 62 los alumnos que finalmente participaron. En total, suman una muestra de 108 participantes.

Como estrategia de recogida de datos se ha empleado la entrevista semiabierta guiada por un cuestionario previamente validado por juicio de expertos, con un grado de acuerdo del 98\% en torno a aspectos de pertinencia, claridad, relevancia y compromiso de

3- Cálculo realizado con un monto de error del $10 \%$, por tanto de confianza del $90 \%$. 
las cuestiones sobre diversidad cultural en las aulas y centros. Las dimensiones concretas pueden observarse sintéticamente en la tabla de respuestas del apartado siguiente. Las entrevistas fueron debidamente grabadas en audio, en un contexto distendido y familiar para los participantes, y fielmente transcritas a posteriori.

Respecto del análisis de datos se ha procedido en un primer momento mediante análisis de contenido temático basado en categorías, por varios investigadores, a través de la codificación a posteriori de las declaraciones de los entrevistados y su reagrupaciónreducción en metacategorías, lo cual nos permitirá dibujar el perfil de cada entrevistado primero y cada colectivo después. Se han considerado las respuestas mayoritarias, recurriendo a los porcentajes para poder contrastar los casos, dado que el tamaño muestral de los mismos es diferente. Se han agrupado las respuestas sobre dimensiones en función de la coincidencia de las mismas, según porcentajes.

Una vez aplicada esta técnica cualitativa, se ha procedido de modo cuantitativo. Se han calculado diferencias de los sujetos de un mismo caso (intracaso), de acuerdo con sus rasgos esenciales: edad, sexo, antigüedad (para el caso exclusivo de los docentes: menos de 1 sexenio, entre 1 y 2 sexenios, más de 2 sexenios), nivel de estudios (para el caso de los progenitores: primarios, medios y superiores), curso ( $3^{\circ} \mathrm{ESO}^{4}, 4^{\circ} \mathrm{ESO}, 1^{\circ}$ Bachiller, $2^{\circ}$ Bachiller), etapa (ESO o Bachiller), zona (rural o urbana), centro (Francisco Ayala, Alonso Cano o Montes Orientales 5 ). Para ello se ha utilizado la prueba de chi cuadrado exacta, con un nivel de significación de $\mathrm{p}=0,05$. Posteriormente, se ha procedido a identificar las diferencias entre los 3 colectivos, para lo cual se ha recurrido al ANOVA aceptando un porcentaje de error también de 5\% $(\mathrm{p}=0,05)$. Cuando no se han hallado diferencias significativas entre los casos, se ha calculado el coeficiente de correlación rho de Spearman ${ }^{6}$, para valorar la relación que se establece entre casos, con objeto de detectar la concordancia interpretada como la influencia de unos casos en otros, con idéntico nivel de significación estadística. Esta última, la influencia, será ratificada por el coeficiente de determinación $R^{2}$, obtenido a partir del anterior, y la potencia predictiva o estimativa $P$ de la regresión, junto a la ecuación de regresión global, tomando para ello los valores en unos perfiles actitudinales (principalmente en el caso de hijos o alumnos) como variables dependientes potencialmente determinadas por los valores de los otros respectivamente (profesores y progenitores).

\section{Resultados}

Se presenta, en primer lugar, la agrupación de respuestas por categorías de las mismas y según cada colectivo. A continuación, los resultados del análisis siguen la estructura del grado de concordancia hallado en las respuestas mayoritarias, a través de los porcentajes de aparición, lo que responde al objetivo de dibujar el perfil-patrón perceptivo-actitudinal de cada uno. Complementariamente, se busca el respaldo estadístico mediante el cálculo de sus diferencias o ausencia de ellas, y en este último caso, la correlación entre colectivos, que responde a la influencia de unos en otros.

4- Educación Secundaria Obligatoria. Edades comprendidas en ESO entre 12 y 16 años (ej. $3^{\circ}$ de ESO= entre 14 y 15, aproximadamente) y en Bachiller entre 16 y 18 años (ej. $1^{\circ}$ de Bachiller: entre 16 y 17 aproximadamente).

5- Todos los centros están en Granada, España.

6- Se ha optado por el coeficiente de Spearman, en lugar de otros, como el de Pearson (aunque los valores no varían significativamente, en este caso), debido a que la naturaleza de los datos aconseja más el empleo de aquél. 
Tabla 1- Agrupación y tendencia de las respuestas categorizadas de alumnado y familias

\begin{tabular}{|c|c|c|c|c|}
\hline Ítem & Categorías / Porcentajes & Docentes & Familias & Alumnos \\
\hline \multirow{3}{*}{ 1. Origen alumnado diverso } & De la zona & 37,5 & 59,1 & 57,4 \\
\hline & Multiculturalidad & 58,3 & 36,4 & 36,1 \\
\hline & De etnia gitanas & 4,2 & 4,5 & 6,6 \\
\hline \multirow{4}{*}{ 2. Conocimiento otras culturas } & Ninguno & 0 & 42,9 & 8,6 \\
\hline & Escaso y general & 95,8 & 28,6 & 89,7 \\
\hline & Medio & 0 & 23,8 & 1,7 \\
\hline & Bastante & 4,2 & 4,8 & 0 \\
\hline \multirow{3}{*}{ 3. Conducta-Rendimiento de alumnado diverso } & Malos & 60 & 52,6 & 6,7 \\
\hline & Normales & 15 & 36,8 & 40 \\
\hline & Buenos & 25 & 10,5 & 53,3 \\
\hline \multirow{4}{*}{ 4. Relaciones interculturales del alumnado } & Malas & 12,5 & 4,5 & 6,8 \\
\hline & Regulares & 25 & 4,5 & 20,3 \\
\hline & Normales & 50 & 31,8 & 11,9 \\
\hline & Buenas & 12,5 & 59,1 & 61 \\
\hline \multirow{4}{*}{ 5. Creencias y tradiciones multiculturales en aula } & Sí & 62,5 & 47,6 & 26,7 \\
\hline & Casos particulares & 33,3 & 4,8 & 0 \\
\hline & A veces & 0 & 9,5 & 41,7 \\
\hline & No & 4,2 & 38,1 & 31,7 \\
\hline \multirow{3}{*}{ 6. Dificultades por pluralismo cultural } & Ninguna & 58,3 & 40,9 & 16,9 \\
\hline & Algunas & 0 & 13,6 & 42,4 \\
\hline & Sí & 41,7 & 45,5 & 40,7 \\
\hline \multirow{3}{*}{ 7. Ventajas por pluralismo cultural } & Ventajas colectivas (todos) & 47,8 & 13,6 & 10 \\
\hline & Enriquecimiento personal & 47,8 & 81,8 & 83,3 \\
\hline & Ninguna ventaja (contrario) & 4,3 & 4,5 & 6,7 \\
\hline \multirow{2}{*}{ 8. Función escolar frente a diversidad cultural } & Enseñanza & 62,5 & 57,1 & 42 \\
\hline & Adaptación e integración & 37,5 & 42,9 & 58 \\
\hline \multirow{3}{*}{ 9. Objetivo con alumnado diverso } & Integración & 91,7 & 48,1 & 57,4 \\
\hline & Respeto & 8,3 & 28,1 & 0 \\
\hline & Aprendizaje & 0 & 23,8 & 42,6 \\
\hline \multirow{3}{*}{ 10. Objetivo con sus familias } & Participar & 75 & 30 & 39 \\
\hline & Integración & 16,7 & 60 & 49,2 \\
\hline & Conocer centro & 8,3 & 10 & 11,8 \\
\hline \multirow{3}{*}{ 11. Formación docente } & Suficiente (experiencia) & 58,3 & 55,6 & 55 \\
\hline & Regular & 4,2 & 22,2 & 25 \\
\hline & Insuficiente & 37,5 & 22,2 & 20 \\
\hline \multirow{4}{*}{ 12. Contenido de preparación docente } & Formación & 81 & 33,3 & 35 \\
\hline & Conocer culturas & 9,5 & 47,6 & 48,3 \\
\hline & Atención familias-alumnos & 9,5 & 14,3 & 16,7 \\
\hline & Ninguna & 0 & 4,8 & 0 \\
\hline \multirow{3}{*}{$\begin{array}{l}\text { 13. Diferencias de participación de familias en centro } \\
\text { según cultura }\end{array}$} & Sin diferencias & 66,7 & 45 & 53,4 \\
\hline & Con diferencias & 25 & 35 & 44,8 \\
\hline & No participan & 8,3 & 20 & 1,8 \\
\hline \multirow{3}{*}{ 14. Relación profesorado-familias } & Buena & 70,8 & 83,3 & 78,6 \\
\hline & Escasa & 10 & 5,6 & 2,4 \\
\hline & Ninguna & 19,2 & 11,1 & 19 \\
\hline \multirow{2}{*}{ 15. Dificultades relación anterior } & No & 91,7 & 100 & 70 \\
\hline & Sí & 8,3 & 0 & 30 \\
\hline \multirow{4}{*}{ 16. Resolución problemas interculturales } & Diálogo & 50 & 62,5 & 56,3 \\
\hline & Participación & 43,8 & 18,8 & 0 \\
\hline & Integración & 6,3 & 6,3 & 40,6 \\
\hline & Irresolubles & 0 & 12,5 & 3,1 \\
\hline \multirow{3}{*}{ 17. Tratamiento educativo a diversidad en aulas } & Riqueza & 26,1 & 19 & 37,5 \\
\hline & Integración & 0 & 62 & 44,6 \\
\hline & Aprendizaje & 73,9 & 19 & 17,9 \\
\hline \multirow{3}{*}{ 18. Observaciones } & Integración & 16,7 & 55,5 & 66,7 \\
\hline & Igualdad & 83,3 & 22,2 & 22,2 \\
\hline & Prejuicios & 0 & 22,2 & 11,1 \\
\hline
\end{tabular}

Fuente: Elaboración propia. 


\section{Dimensiones perceptivo-actitudinales semejantes de docentes-familias-discentes ante la diversidad}

Tomando como base las declaraciones en las dimensiones más frecuentes y coincidentes de los tres colectivos, que reflejan alto grado de unanimidad, puede dibujarse un perfil claro e inequívoco de sus actitudes y percepciones. Sorprendentemente, esto solo ocurre en una tercera parte de las dimensiones cuestionadas (33,3\%); como se observa en la tabla y se analiza seguidamente.

Gráfico 1- Datos por colectivos y dimensiones donde existe un perfil semejante

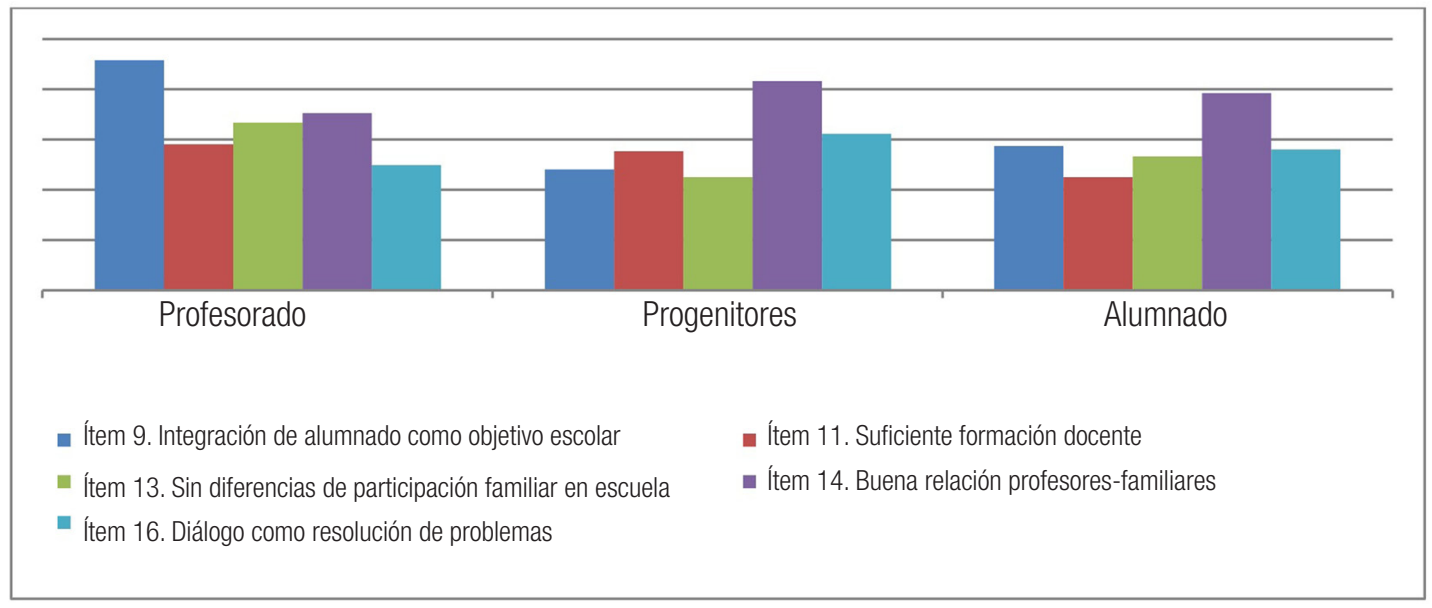

Fuente: Elaboración propia.

- El objetivo básico a conseguir con el alumnado de culturas diferentes a la mayoritaria dentro del aula ha de ser la integración. Es una apuesta decidida del profesorado $(91,7 \%)$, que secundan los padres/madres y sus hijos, aunque con menor intensidad (48,1 y 57,4\%, respectivamente), entre los que se encuentran otras respuestas diferentes (según zona-centro en el caso de los progenitores, y zona-centro, edad y curso en el caso del alumnado) que apuntan también hacia el aprendizaje y el respeto. Precisamente, a pesar de la coincidencia en las respuestas mayoritarias, las diferencias entre todas sus respuestas alcanzan el grado de significación estadística ${ }^{7}$, como consecuencia de la diferencia entre profesores y progenitores y profesores y alumnos ${ }^{8}$; pero no entre progenitores y alumnos, donde las diferencias no alcanzan significatividad estadística9.

- No cabe duda de que piensan que los docentes disponen de preparación suficiente, lo que es apoyado por más del 50\% de cada caso, siendo los docentes los más críticos consigo mismos, y los progenitores los que más diferencias intracaso presentan (según sexo, centro y curso), seguidos del profesorado que diferencian sus respuestas según la zona. Además, calculadas las diferencias globales en todas sus respuestas, el valor no

\footnotetext{
7- Para un $p$-valor $=0,01$.

8- $p=0,01$ y 0,00 , respectivamente.

9- $p=1$.
} 
alcanza la significatividad exigida entre los grupos ${ }^{10}$, luego no se aprecian discrepancias intercasos.

- No se declaran diferencias en la participación en la escuela de unas familias y otras de acuerdo con su origen cultural. Ahora bien, precisamente las familias, que son las más implicadas en esta cuestión, manifiestan menos contundencia en sus respuestas, esto es, mientras que para el resto de colectivos la afirmación anterior supera el 50\% de las respuestas, en este caso no lo supera, solo un 45\% opina sobre la ausencia de diferencia y, además, otro 35\% opina que sí las hay, lo que aparece corroborado por el $44,8 \%$ del alumnado. Las divergencias dentro de estos colectivos se deben a la zona, al igual que las que se producen, aunque menos, entre el profesorado. Además de la zona, los alumnos varían su respuesta en función del centro, lo que está, en cierta medida, relacionado con la zona. Aun así no existen diferencias significativas entre las respuestas totales de los tres $\operatorname{casos}^{11}$.

- Un alto grado de acuerdo se detecta respecto a la buena relación existente entre profesorado y familias, en todos los casos supera el 70\% esta opción. Cierta diferencia significativa se aprecia entre las respuestas del profesorado, respecto de la zona-centro, y en el alumnado por las variables zona-centro, principalmente, además del sexo y curso. No son significativas las diferentes entre las respuestas ofrecidas por cada colectivo ${ }^{12}$.

- La resolución de problemas debe acometerse a través del diálogo, principalmente, lo cual está apoyado por más del 50\% en cada caso; además no genera diferencias significativas entre las variables consideradas. También existe cierto grado de acuerdo entre el resto de las respuestas minoritarias según sus porcentajes, lo que hace que no resulten significativas las diferencias de los distintos casos ${ }^{13}$.

- Por último, predomina en todas las declaraciones la ausencia de dificultades en las relaciones entre profesores y las familias. Lo corroboran los implicados con alto porcentaje (91,7 y 100\%, respectivamente) y asimismo lo perciben los alumnos, sin mostrar diferencias internas dentro de los casos. No obstante, en esta ocasión sí existen diferencias en el resto de las respuestas entre profesores y alumnos, así como entre progenitores y alumnos $^{14}$, aunque no entre profesores y progenitores ${ }^{15}$.

Tras la ausencia generalizada de diferencias significativas entre las respuestas de los tres casos a las dimensiones anteriores (excepto diferencias parciales entre docentes y profesores y docente y discentes en la primera y en la última cuestión), la correlación calculada entre ellas es considerable. Entre docentes y progenitores, así como entre docentes $\mathrm{y}$ alumnos se produce una correlación de tipo directa e intensidad media ( $\mathrm{rho}=0,65^{16} \mathrm{y}$ $\left.0,58^{17}\right)$. Más intensa resultó la correlación, también directa, entre progenitores y alumnado $\left(\right.$ rho $\left.=0,75^{18}\right)$, hasta el punto de considerarse relativamente alta (dada la ausencia absoluta

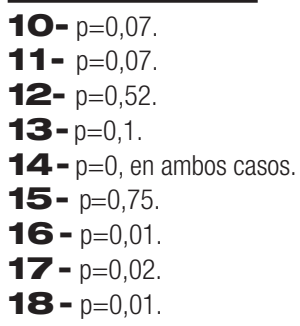


de discrepancias entre ellos), en lugar de media como los valores anteriores. En efecto, acudiendo al coeficiente $\mathrm{R}^{2}$ de determinación apunta valores bajos para los primeros casos $(\mathrm{R} 2=0,42$ y 0,34$)$, sin embargo, supera el 0,5 en el caso último $(\mathrm{R} 2=0,56)$, lo que apunta hacia una determinación media.

\section{Dimensiones perceptivo-actitudinales comunes de progenitores-hijos ante la diversidad}

Estos datos unidos a los anteriores podrian reinterpretarse como la influencia de las actitudes y percepciones de los progenitores en sus descendientes. En esta ocasión, se presenta una proporción ligeramente superior de acuerdo sobre la respuesta a la anterior $(38,9 \%)$ en las dimensiones siguientes (ítem 1, 4, 7, 10, 12, 17 y 18), en las que se aprecian además diferencias entre ellos y los docentes, como se observa en el gráfico que sigue:

Gráfico 2- Datos por colectivos y dimensiones semejante entre progenitores y alumnado

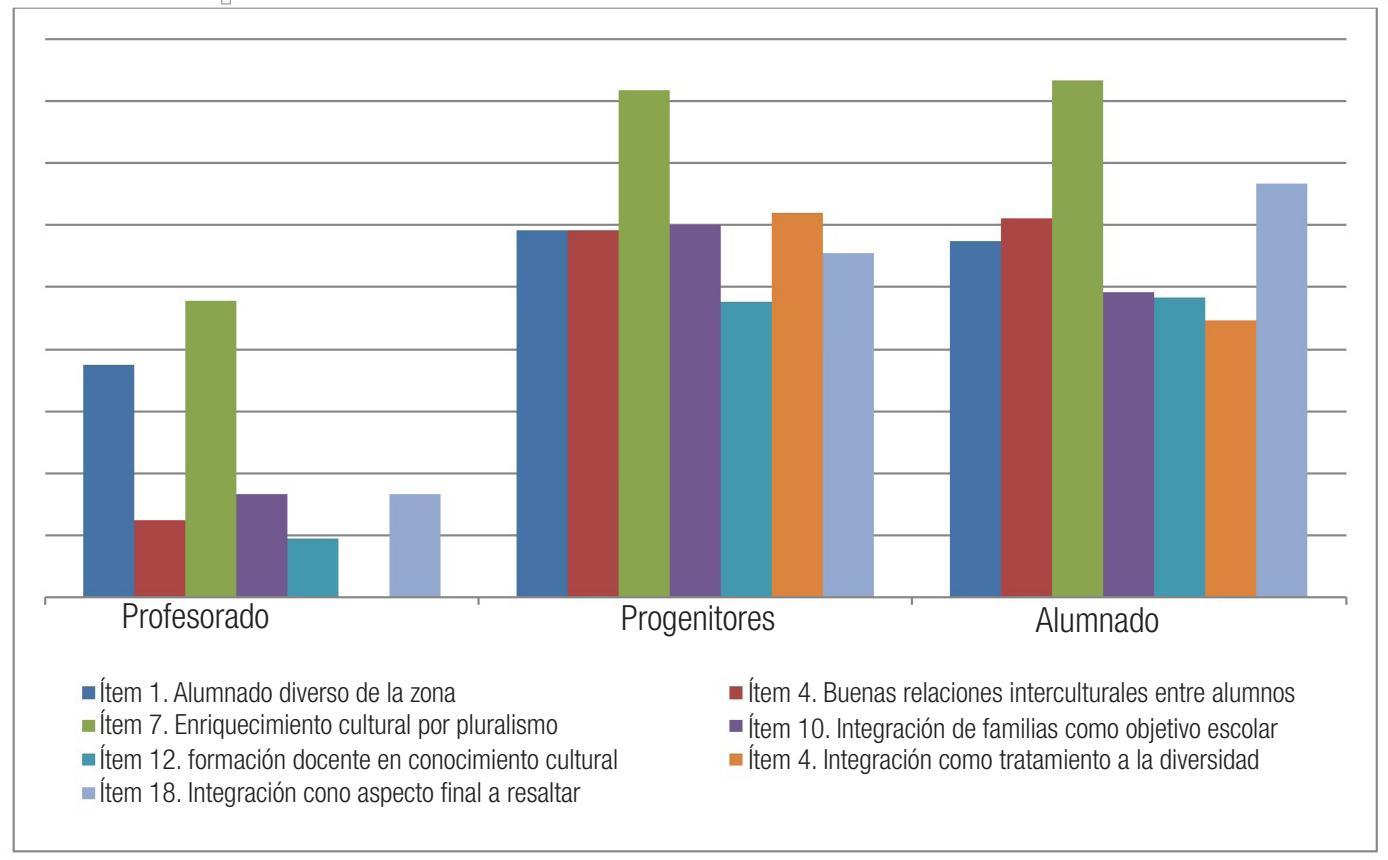

Fuente: Elaboración propia.

- Progenitores e hijos mayoritariamente consideran al alumnado de otras culturas como de la zona, con cierto arraigo ${ }^{19}$, mientras que los docentes siguen considerándolos como multicultural foráneo, ávidos de identidad y arraigo. Como las familias presentan ciertas diferencias en esta percepción de acuerdo con la zona-centro y el curso, los alumnos las reproducen, según zona-centro, curso y edad. También en el profesorado el curso establece diferencias significativas. Parece apuntar que los participantes de cursos

$19-p=0.97$ 
superiores cambian el concepto del alumnado diverso, quizá porque en estas aulas no obligatorias la diversidad cultural es diferente a las precedentes.

- Las relaciones interculturales entre alumnos son concebidas como buenas por progenitores e hijos, en proporción idéntica, con variaciones ligeramente mayores en el caso de los hijos (edad, zona-centro y curso), como consecuencia quizá de la influencia del profesorado que las considera solo normales. Las diferencias intercasos resultan significativas $^{20}$, lo cual se debe a las discrepancias entre profesores y padres/madres ${ }^{21}$ y entre profesores y alumnos ${ }^{22}$, pero no entre progenitores e hijos ${ }^{23}$.

- Idénticos vuelven a presentarse los porcentajes de respuesta de padres/ madres e hijos respecto de la ventaja que se deriva del pluralismo cultural por cuanto enriquecimiento personal (81,8 y 83\%, respectivamente), de hecho sus diferencias entre respuestas globales no son significativas ${ }^{24}$, a diferencia del profesorado cuyas respuestas se reparten equitativamente $(47,8 \%)$ entre el enriquecimiento personal y las ventajas para el colectivo, resultando significativas las diferencias con respecto tanto a padres/ madres como a alumnado ${ }^{25}$. Las diferencias intracaso solo son significativas en el caso del profesorado de acuerdo con la antigüedad en el puesto de trabajo.

- Progenitores y descendientes muestran respuestas semejantes a la pregunta del objetivo escolar para con las familias, priorizando la integración de las mismas, seguida de la participación. En el caso del alumnado las respuestas según edad, curso y centro difieren significativamente. Las diferencias entre ambos colectivos no son significativas ${ }^{26}$. Los docentes, sin embargo, apuestan más por la segunda opción de los anteriores: la participación de las familias en el centro, aunque se observan diferencias de acuerdo con variables zona-centro y curso. Siendo así, resultan significativas las diferencias entre respuestas docentes y respuestas discentes ${ }^{27}$, pero no alcanza significatividad las diferencias entre respuestas de docentes y progenitores ${ }^{28}$.

- El contenido de la preparación docente necesaria para mejorar la atención a la diversidad cultural se ha de conseguir mediante el conocimiento de las distintas culturas que cohabitan en el aula, según los padres/madres (47,6\%) y sus hijos (48,3\%) (solo difieren las respuestas según la edad de ambos colectivos), mientras que las respuestas de los propios docentes reclaman una mejor formación inicial, que consideran entre insuficiente y alejada de la realidad. Por tanto, en el primer caso las semejanzas superan a las diferencias, mientras que en el último acontece lo contrario ${ }^{29}$.

- Tanto progenitores como descendientes coinciden en resaltar nuevamente la integración como el tratamiento adecuado a la diversidad en las aulas, con ciertas diferencias intracasos según la edad de los primeros y la edad, sexo, curso y centro de los segundos, pero sin diferencias generales intercasos ${ }^{30}$. Y este tratamiento se diferencia del

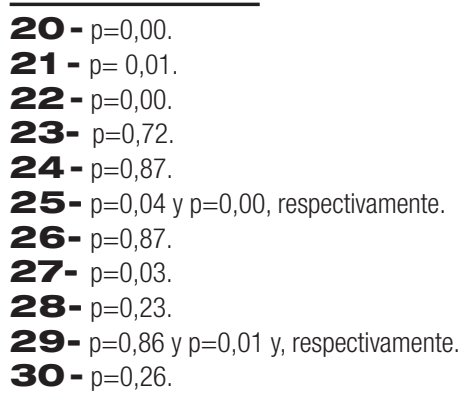


declarado por el profesorado tanto respecto de las familias como del alumnado ${ }^{31}$, que se decanta más hacia el aprendizaje.

- Por último, en la última pregunta sobre observaciones, la integración es lo más reclamado por padres/madres (55,5\%) e hijos (66,7\%), sin diferencias significativas en esa y en el resto de las respuestas minoritarias ${ }^{32}$, mientras los docentes reclaman la igualdad de oportunidades claramente (83,3\%). Y en estas repuestas no existen diferencias significativas ni intracaso ni intercaso ${ }^{33}$.

En efecto, procede calcular las correlaciones, dadas las escasas diferencias entre progenitores y descendientes, mediante el coeficiente de corrección de Spearman. La correlación entre familiares e hijos es directa, muy alta $(r h o=0,86)$ y significativa ${ }^{34}$, así como su determinación $\left(\mathrm{R}^{2}=0,74\right)$. No obstante, se observa que no están relacionadas las respuestas a las anteriores dimensiones entre docentes y familiares, según muestran sus bajos valores $(\mathrm{rho}=0,16$ y 0,26$)$ y su falta de significatividad ${ }^{35}$ y ratifican sus ínfimos valores de regresión $\left(R^{2}=0,03\right.$ y 0,07$)$.

\section{Dimensiones perceptivo-actitudinales coincidentes de profesorado-padres/madres ante la diversidad}

Alejada de los porcentajes de coincidencia anteriores, en concreto con la mitad de peso porcentual (16,7\%), le sigue la semejanza de respuesta frecuente entre profesores y progenitores con diferencias respecto del alumnado, como se observa en la tabla, en las cuestiones 3, 5 y 8, a saber:

Gráfico 3- Datos por colectivos y dimensiones semejantes entre profesorado y progenitores

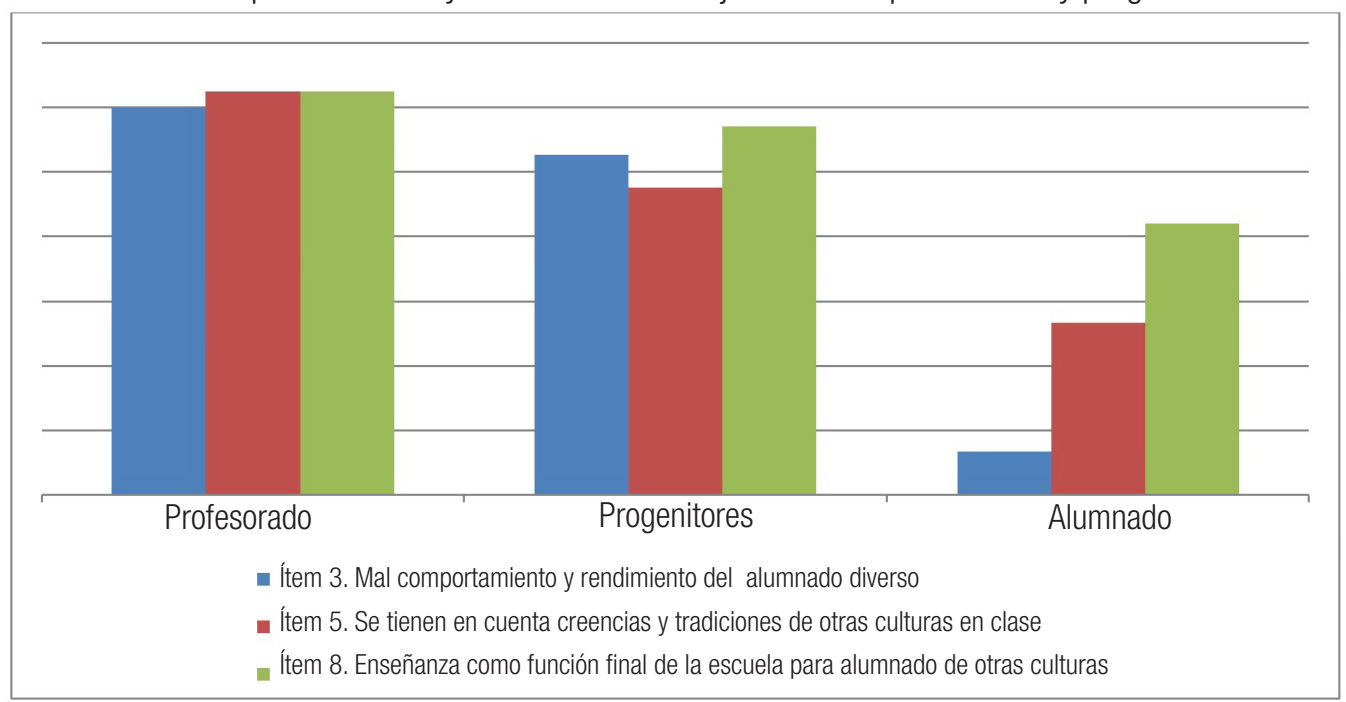

Fuente: Elaboración propia.

\footnotetext{
31- $p=0,01$ y 0,2 , respectivamente.

32- $p=0,04$.

33- $p>0,05$.

34- $p=0,00$.

35- $p=0,59$ y $p=0,29$, respectivamente.
} 
- Tanto profesores como progenitores perciben como malos el comportamiento y rendimiento de alumnos de culturas minoritarias (con leves variaciones según la zonacentro del profesorado y la edad y estudios de los progenitores). En efecto, las divergencias entre sus respuestas globales no son significativas ${ }^{36}$. Los alumnos, sin embargo, creen que son buenas, mostrando más diferencias entre ellos, según la edad, el sexo, el curso y la zona-centro. De hecho, las diferencias entre sus respuestas y las de profesores y progenitores no dejan lugar a dudas en cuanto a significación ${ }^{37}$.

- Igualmente, los adultos entrevistados contestan que se tienen en cuenta las creencias y las costumbres en el aula (currículum intercultural), sobre todo los profesores, entre los que no hay diferencias internas, a diferencia de los progenitores que sí difieren en sus respuestas, de acuerdo con el centro y el nivel de estudios. Entre unos y otros no hay diferencias significativas en sus respuestas ${ }^{38}$. Por el contrario, los alumnos piensan que solo son tenidas en cuenta en ocasiones, aunque sus respuestas se reparten entre otras opciones, como nunca o sí son tenidas en cuenta, en función del sexo, edad, curso y centro. Las diferencias calculadas entre respuestas del alumnado y del profesorado son significativas como lo son, aunque en menor medida, entre alumnado y progenitores ${ }^{39}$.

- Mientras que los adultos piensan que la labor de la escuela es la enseñanza (con leves variaciones según la zona-centro del profesorado y la edad por parte de las familias), los menores apuestan por la adaptación e integración, aunque con diferencias significativas entre sus respuestas, según edad, sexo, curso, zona-centro. No se establecen diferencias significativas entre los adultos y sí entre ellos y los menores investigados ${ }^{40}$.

La correlación entre profesores y padres/madres en las dimensiones anteriores es significativamente intensa y directa $\left(\mathrm{rho}=0,74^{41}\right)$ como consecuencia de la falta de diferencias; mientras que la relación calculada entre profesores y alumnos resulta baja e inversa $(r h o=-0,1)$ y baja y directa $(r h o=0,1)$ entre familiares y alumnos; evidenciando ambos cálculos escasa significatividad ${ }^{42}$. La determinación es notable en el primer caso $\left(R^{2}=0,55\right)$, mientras que es ínfima en las últimas $\left(R^{2}=0,01\right.$, en ambos).

\section{Dimensión perceptivo-actitudinal semejante de docentes-discentes ante la diversidad}

Apenas en la cuestión 2 (5,6\%) se muestra cierta coincidencia entre las respuestas emitidas por los docentes y los discentes que difiere de la de padres/madres. Los docentes $\mathrm{y}$ discentes declaran poseer un conocimiento escaso y general de otras culturas, en un excelso porcentaje de sus respuestas (95,8 y 89,7\%, respectivamente), que no deja lugar a diferencias individuales ${ }^{43}$, por encima del nulo conocimiento declarado por los padres/

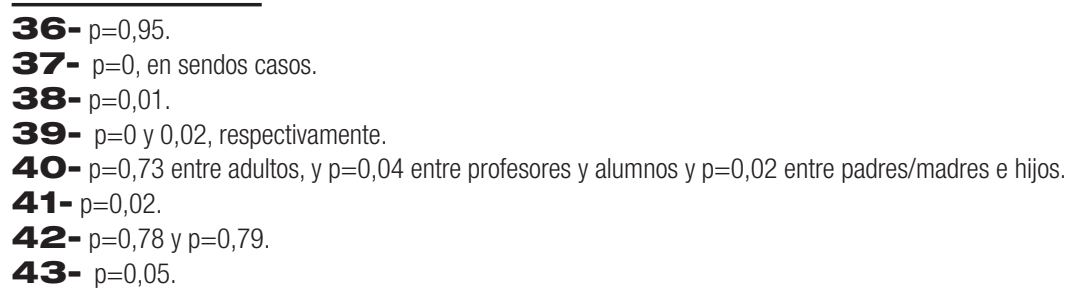


madres $(42,9 \%)$, sin diferencias individuales igualmente. Respecto de la globalidad de las respuestas, no se producen diferencias significativas entre los docentes y discentes ${ }^{44}$ y sí entre cada uno de ellos con padres/madres ${ }^{45}$.

Gráfico 4- Datos por colectivos y dimensión semejante entre docente y discente

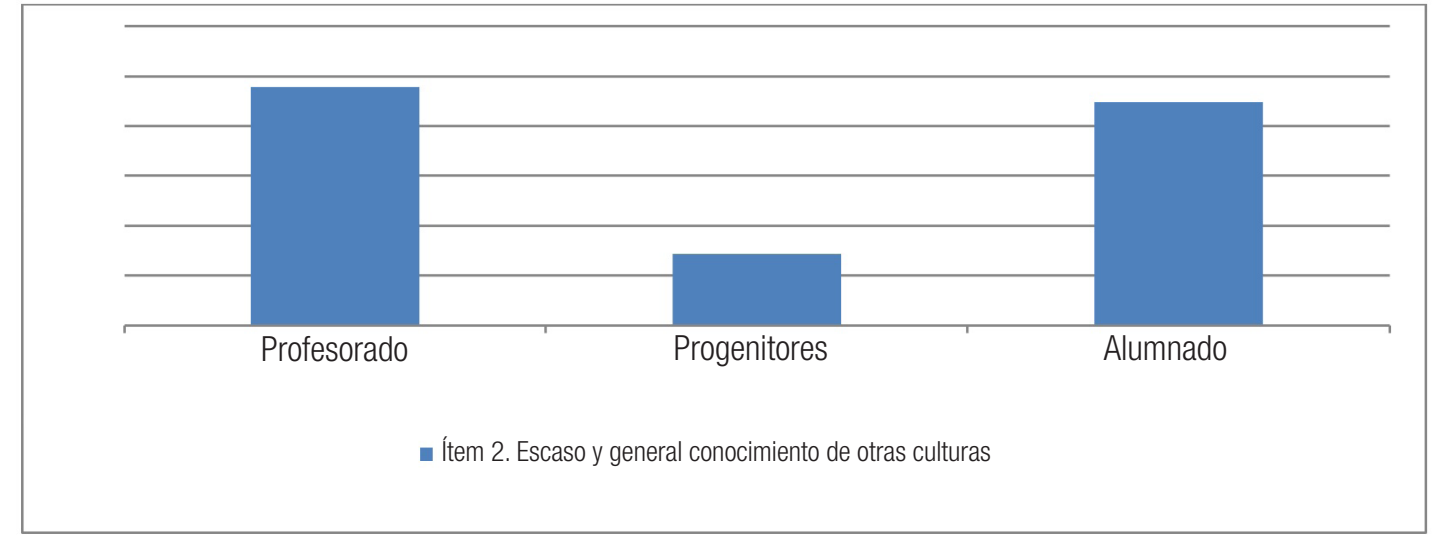

Fuente: Elaboración propia.

La correlación entre docentes y alumnos obtenida para esta dimensión es alta y directa $(r h o=0,9946)$ y la determinación intensa $\left(\mathrm{R}^{2}=0,98\right)$. Sin embargo, la relación establecida entre respuestas de docentes y familiares se presenta baja e inversa ( $\mathrm{rho}=-0,21$; $\left.\mathrm{R}^{2}=0,04\right)$, y entre familias y alumnos baja y directa (rho= 0,$\left.23 ; \mathrm{R}^{2}=0,05\right)$, aunque ambas carecen de significación estadística ${ }^{47}$.

\section{Dimensión perceptivo-actitudinales discrepante entre docentes-familias-discentes ante la diversidad}

Por último, en otra cuestión (6; 5,6\%) las respuestas no coinciden en absoluto entre unos y otros colectivos entrevistados. Los docentes no encuentran mayoritariamente dificultad alguna derivada del pluralismo cultural en las aulas $(58,3 \%)$, aunque existen diferencias según la zona-centro, que hace que un grupo nutrido sí indique su existencia $(41,7 \%)$. Lo contrario ocurre con los familiares; que señalan mayoritariamente dificultades por convivencia multicultural (45,5\%); aunque también aparecen voces contrarias en porcentaje notable (40,9\%). Con tal confusión, es de esperar que los alumnos repartan sus respuestas entre algunas dificultades (42,4\%) y bastantes dificultades (40,7\%), siendo centro y curso los responsables de tales diferencias. Diferencias que alcanza la significación estadística $^{48}$, siendo porcentualmente más cercanas las declaraciones de progenitores e

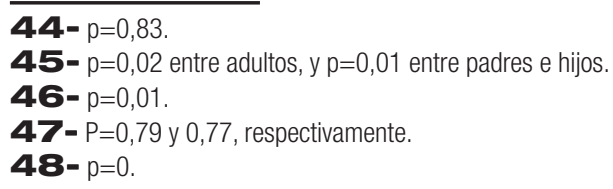


hijos que las de profesorado y progenitores y profesorado y alumnos, lo que produce que la correlación carezca de significación estadística ${ }^{49}$.

Gráfico 5- Datos por colectivos v dimensiones discrepantes declaradas por ellos

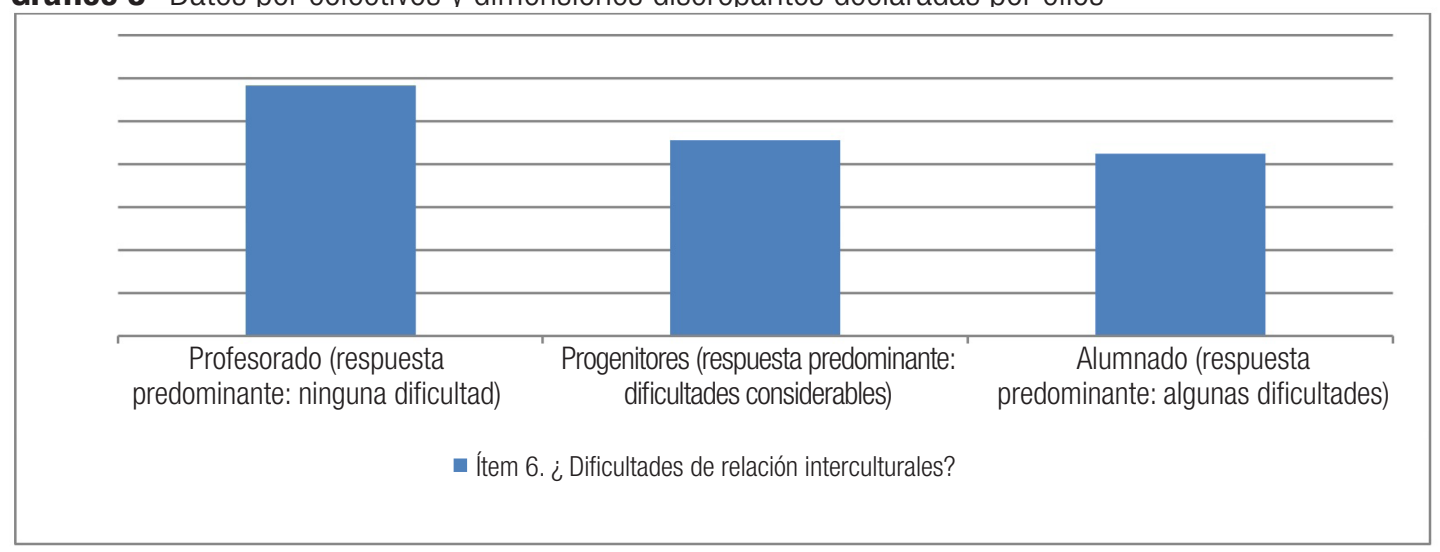

Fuente: Elaboración propia.

\section{Discusión de resultados}

En relación con el profesorado, los peores comportamientos y rendimientos percibidos del alumnado de otras culturas son corroborados por Salas y otros autores (2012), lo cual deviene en problemas de convivencia que son resaltadas en el presente estudio, también advertidas por INJUVE (2008) y otros contextos (SETTON, 2017), y de rendimiento por desconocimiento del idioma en el caso de inmigrantes (MOLINER; MOLINER, 2010), absentismo por desvinculación familiar (FRANZÉ, 2008) y deprivación sociofamiliar (AGUADO et al., 2003) en el caso de gitanos. No obstante, en este último estudio los autores concluyeron que los docentes investigados conciben las relaciones como buenas tanto entre ellos y el alumnado como entre el alumnado, como en el caso de esta investigación. Intxausti, Etxebarria y Joaristi (2014) descubren que las expectativas del profesorado sobre relaciones entre alumnos inmigrantes es que se relacionen entre ello, y no con alumnos de otras culturas; mientras que Santos, Lorenzo y Priegue (2011) añaden que estas expectativas varían según la procedencia cultural. Y Gómez (2015) alerta que de ellas pueden surgir conflictos e incluso episodios violentos, comunes en la actualidad, siendo el alumnado inmigrante más vulnerable y más víctima que acosador. Con todo, se propone la necesidad de una mejor-mayor integración del alumnado sin olvidar su aprendizaje y participación familiar (a pesar de que la relación sea buena); puestas de manifiesto en esta investigación como objetivos escolares prioritarios para con el alumnado y con sus familiares.

Cuando se invita a cuestionar su propia formación docente, rara vez se concluye sobre su idoneidad, encontrando aspectos necesitados y déficits en: a) actitudes docente adecuadas para enseñar en contextos multiculturales (LEIVA 2008; 2012; MERINO; RUIZ,

$\mathbf{4 9}$ - $p=0,67,0,45$ y 0,67 . 
2005; MERINO; LEIVA, 2007; SALAS et al., 2012); b) expectativas para alumnos de culturas minoritarias (INTXAUSTI; ETXEBARRIA; JOARISTI, 2014); c) información sobre culturas minoritarias (AGUADO et al., 2003) y características del alumnado inmigrante (INTXAUSTI; ETXEBARRIA; JOARISTI, 2014) o gitano (MOLINER; MOLINER, 2010), d) relaciones con las familias, para su conocimiento y participación: “[...] ver y acercar a las familias a la escuela” (FRANZÉ, 2008, p. 129); e) formación general tanto inicial como permanente (GÓMEZ, 2015; LEIVA, 2008, 2010). Todos ellos basados exclusivamente en la experiencia y a distancia de la altura del escenario actual. En esta investigación se oyeron voces críticas sobre su formación (aunque con predominio leve de su suficiencia), junto al escaso conocimiento cultural y bajas expectativas conductuales y de rendimiento, reclamantes de formación pedagógica e información cultural. Ahora bien, intentan tener en cuenta las creencias y tradiciones de otras culturas, en la medida de sus competencias y conocimientos limitados autodeclarados, lo que ha avanzado desde otras investigaciones pasadas donde se negaba su preocupación por este asunto y por desarrollar un currículo intercultural en una escuela multicultural, al punto que se execraba el intento de hacer invisibles las diferencias culturales como forma de entender la igualdad de oportunidades, lo cual amenaza el tratamiento equitativo y justo de la diversidad, que es diferente del igualitario para todos.

De parte del alumno, las investigaciones temáticamente afines a ésta, obtienen resultados negativos: los alumnos muestran actitudes contrarias a la integración (BARRENA, 2014), incluso rechazan a los inmigrantes (DÍAZ, 2008) e incluso piensan que deberían ser expulsados si no disponen de papeles por suponer a estas alturas de Secundaria una amenaza para el acceso al mercado laboral (CALVO, 2008), por lo que Leiva (2013) propone aumentar y mejorar las relaciones interpersonales en contextos interculturales. Lo cual contradice los hallazgos de esta investigación que pone de manifiesto las buenas relaciones interculturales del alumnado así como la ausencia o escasez de dificultades, incluso resaltando las ventajas para el enriquecimiento personal, aunque se resalten diferencias de tipo religioso e idiomáticas y en menor medida de costumbres, como en otras investigaciones (AGUADO et al., 2003).

Sobre la dimensión familiar, se ha resaltado la necesidad de mayor participación, que ha de fomentarse desde la institución escolar (SALAS et al., 2012; SANTOS; LORENZ0; PRIEGUE, 2011), lo cual es congruente con lo obtenido en la presente investigación. Incrementar esfuerzos personales si fuese preciso (ETXEBARRIA; INTXAUSTI; JOARISTI, 2013), con mediadores (AGUADO et al., 2003) o a través de las Asociaciones de Padres y Madres (GARRETA, 2008). También con nuevos espacios de relación y clima de apoyo por parte de la institución escolar (LEIVA; ESCARBAJAL, 2011). Ello responde a que los centros no satisfacen las demandas de las familias de culturas minoritarias así como la problemática inherente a las relaciones interculturales (SALAS et al., 2012), lo cual también ha sido puesto de manifiesto en esta investigación. En definitiva, se refuerza el hallazgo previo de que las familias admiten que participan poco (AGUADO et al., 2003; ETXEBARRIA; INTXAUSTI, 2013; GARRETA, 2008; INTXAUSTI; ETXEBARRIA; JOARISTI, 2014; SANTOS; LORENZO, 2009; SANTOS; LORENZO; PRIEGUE, 2011). Según Aguado y otros (2003), los familiares excusaron su falta de participación por su escasa contribución 
al hecho educativo, así como por no interferir en la tarea docente que es buena, porque son buenos profesionales y no por malas relaciones con ellos. Ambas justificaciones coinciden con lo obtenido en esta investigación con familias mayoritarias. Según el estudio de Etxebarria y otros autores (2014) existe una separación entre la Escuela como lugar de aprendizaje y el hogar como motivador y transmisor de valores. Los datos de la investigación presente también apuntan la labor de enseñanza por parte de los centros docentes, aunque se compagina con la integración. De otra parte, la investigación de Santana, Feliciano y Jiménez (2016) advierte el menor apoyo y expectativas por parte de los padres para que ellos continúen sus estudios. Es lo que se ha denominado deprivación cultural que ha sido puesto de relieve en otros estudios que lo critican como origen exclusivo de fracaso escolar potencial, reclamando las cotas de responsabilidad del centro educativo y del profesorado (RUIZ; MERINO, 2009).

Intxausti, Etxebarria y Joaristi (2014) combinan y contrastan los datos obtenidos por docentes y familias respecto de las relaciones sociales del alumnado en circunstancias de convivencia intercultural. Ponen de manifiesto que las expectativas del profesorado son peores que las de familiares, igual que acontece en esta ocasión, donde las peores conductas y rendimientos son puestos de manifiesto por el profesorado. Ello sin duda afecta al resto de agentes, en especial al alumnado, como demuestra Merino (2005) sobre su autoconcepto y agresividad. Solo el estudio de Aguado y otros autores (2003) incluye los tres colectivos: profesores, progenitores y alumnos, aunque no desde la perspectiva del contraste como se ha realizado en esta ocasión sino para proponer realmente cambios demandados por todos los miembros de tal suerte que realmente pueda hablarse de escuela y currículo intercultural, que a juicio de los autores en la actualidad no es más que una desiderata.

\section{Conclusiones}

Existen perfiles perceptivo-actitudinales de los distintos casos y diferencias entre ellos, aunque también cierta similitud que hace sospechar alguna influencia de unos en otros (cf. Gráfico 6):

- La coincidencia absoluta entre ellos se produce únicamente en el 33,3\% de sus respuestas frente a la discrepancia absoluta que solo se presenta en un 5,6\%.

- A lo anterior, cabe añadir la coincidencia de respuestas que dibujan los perfiles de progenitores e hijos, lo que supone un 38,9\%, que sumado al porcentaje de coincidencia absoluta anterior asciende al 72,2\%. La influencia del perfil de padres en descendientes en estas dimensiones queda corroborada por su correlación de respuestas: alta-directa $(\mathrm{rho}=0,80)$. Le corresponde un coeficiente de determinación medio de $\mathrm{R}^{2}=0,64$.

- Le sigue la coincidencia entre profesores y progenitores, 16,7\%, añadiendo la cantidad anterior de acuerdo absoluto alcanza el 50\%. Entre estas respuestas similares se ratifica la correlación alta-directa $(\mathrm{rho}=0,73)$ pero con un coeficiente de determinación menor $\left(\mathrm{R}^{2}=0,5\right)$.

- Por último, en orden prevalente de concordancia, se encuentran las respuestas entre profesores y alumnos, apenas un 5,6\% que unido al acuerdo global lo sitúa 
en $38,9 \%$. Entre las pocas dimensiones coincidentes entre docentes y discentes se obtiene una correlación y determinación idénticas a las anteriores.

Gráfico 6- Datos por colectivos y dimensiones donde existe un perfil semejante
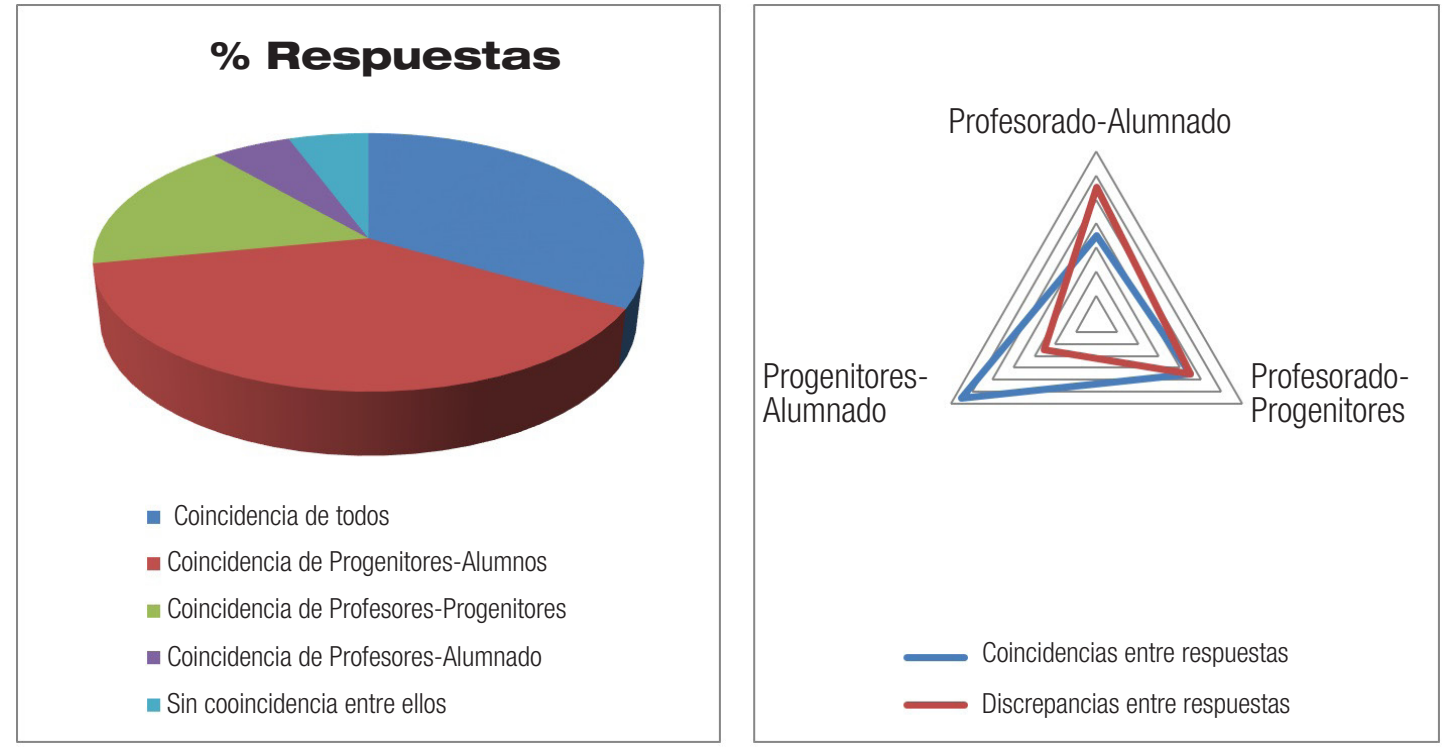

Fuente: Elaboración propia.

Ahora bien, calculado el coeficiente de correlación (de Spearman) sobre todas respuestas por pares de colectivos se demostró que solo entre progenitores y alumnos se obtuvo una correlación total de signo positivo e intensidad media-alta (rho $=0,66$ ) aunque no se puede afirmar lo mismo respecto de la determinación $\left(\mathrm{R}^{2}=0,44\right)$, y en el resto de emparejamientos la correlación resultó ser directa y baja (en torno al rho=0,4 en ambos casos: profesores-progenitores y profesores-alumnos) y más aún su determinación $\left(\mathrm{R}^{2}=0,19\right.$ y 0,15 , respectivamente), con un p-valor que garantiza la significación estadística ${ }^{50}$. Finalmente, se ha realizado un análisis de regresión para estimar la posible influencia de las respuestas del profesorado y de los progenitores en las respuestas del alumnado (Cfr. Gráfico 7). En el primer caso, influencia de docentes en discentes la potencia predictiva ha resultado relativamente baja (PDocentes $\rightarrow$ Discentes $=0,17^{51}$ ) mientras que la influencia estimativa de progenitores en hijos ha resultado media (PProgenitores $\rightarrow$ Descendientes $=0,46^{52}$ ), siendo las ecuaciones de regresión obtenidas para cada caso las que se siguen, apuntando una mayor distancia de respuestas entre profesores y alumnos que entre padres/madres y sus hijos.

$$
\begin{aligned}
& \text { Respuestas-Alumnado }=20,83+0,34 \text { Respuestas-Profesorado } \\
& \text { Respuestas-Alumnado }=9,32+0,71 \text { Respuestas-Progenitores }
\end{aligned}
$$

\footnotetext{
50- $p=0,00,0,01$ y 0,02 , respectivamente.

51- $p=0,01$

52- $p=0,00$.
} 
Gráfico 7- Correlaciones globales entre colectivos de progenitores, docentes y discentes

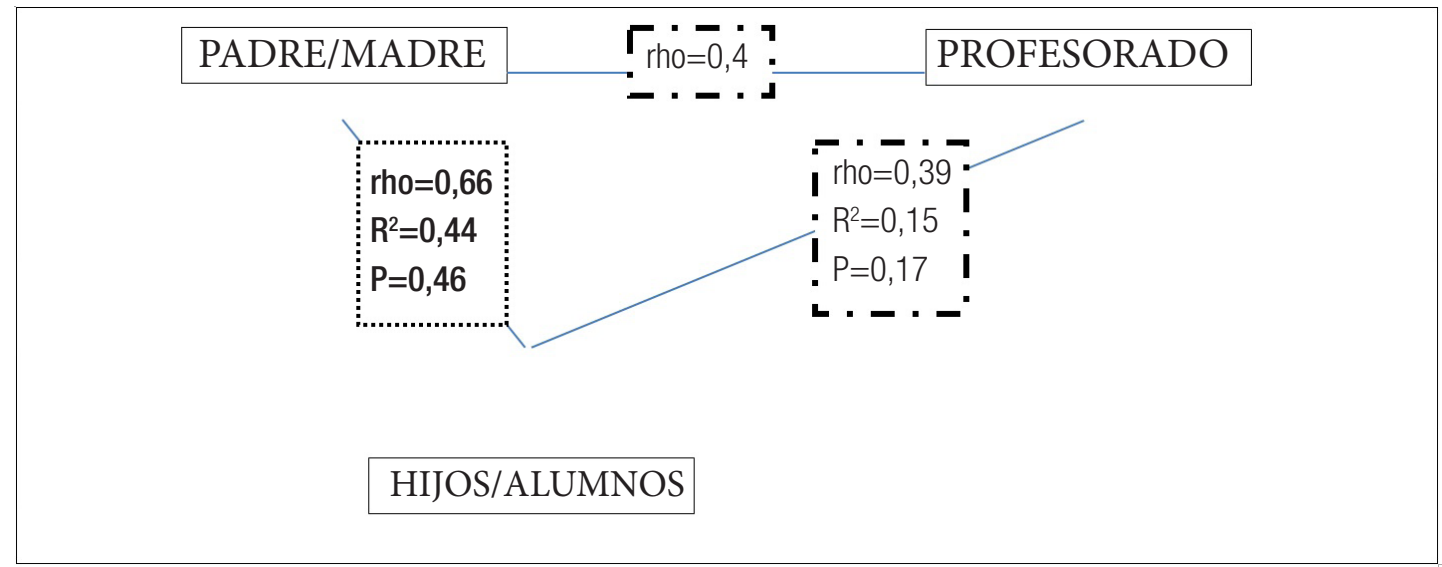

Rho = coeficiente de correlación de Spearman. $\mathrm{R}^{2}=$ coeficiente de determinación. $\mathrm{P}=$ potencia de predicción de la regresión.

Fuente: Elaboración propia.

En definitiva, cabe confirmar la presencia de patrones perceptivo-actitudinales para los colectivos entrevistados, con alto grado de acuerdo interno de cada uno de ellos, si bien significativamente menor entre el sector del alumnado. Al contrario, no puede asegurarse la influencia o determinación entre ellos, aunque entre progenitores y alumnado se producen ciertas confluencias y coincidencias que, en ocasiones, alcanzan una correlación intensa y una determinación considerable, unida a una correlación media en el total de las cuestiones analizadas. Pero en este último caso no tanto en cuanto a su determinación ni en su regresión o estimación, sobre todo cuando se realizan los cálculos con los datos globales de las dimensiones analizadas.

El resto de confluencias entre colectivos es aún menor, entre docentes y discentes casi testimonial, como se desprende de su coeficiente de determinación y de estimación. Ello confirma la preocupación planteada en el problema inicial de la investigación, acerca de que la falta de confluencias perceptivo-actitudinales ante la diversidad cultural entre progenitores y profesores confunde al adolescente en su propia configuración perceptivoactitudinal, abriendo la puerta a la influencia de otros agentes socializadores (grupos de amigos, medios de comunicación, Internet, etc.).

La investigación presentada es descriptiva, muy localizada en un contexto y sujetos determinados, así que su generalización no queda garantizada por el tipo de diseño y método de análisis. Se trata de una primera aproximación en el campo de los perfiles perceptivoactitudinales de los colectivos entrevistados, así como el posible calado de padres/madres, por un lado, y docentes, por otro, en sus hijos/alumnos, respectivamente, como colectivo más influenciable, con intención de detectar divergencias entre ellos que puedan generar confusión y búsqueda inapropiada. Sobre la base de los resultados alcanzados, así como de las limitaciones plasmadas, no cabe duda de que se debe proseguir el camino emprendido, en perspectiva bifronte. Una, en la práctica de los centros, mediante el desarrollo de actividades conjuntas, jornadas de convivencia y organizaciones institucionales que 
conduzcan al aunamiento de actitudes de los colectivos conformantes del estudio, incluso otros de la comunidad educativa: demás profesionales y directivos, demás familiares, grupos de iguales, medios de comunicación, organizaciones no gubernamentales y gubernamentales orientadas a grupos minoritarios para que dejen de ser minoritarizados, incluso políticas con decisiones sobre estos grupos; sin olvidar los sujetos que no han sido objeto de este estudio: alumnado y familiares de culturas minoritarias. Y otra, perpetuando la investigación en el campo que aporte más conocimiento para el corpus teórico del mismo, ampliando contextos, sujetos, características de unos y otros, variando tanto el diseño como el método así como los instrumentos de recogida y análisis de los datos, de tal arte que nos permita alcanzar nuevos retos acerca de patrones perceptivos-actitudinales ante la alteridad, la influencia de nuevos patrones (grupos de iguales, medios de comunicación) en alumnos, así como la manera más apropiada de abordar la optimización de unos, los patrones, y otra, su influencia.

\section{Referencias}

AGUAD0, Teresa et al. Educación intercultural en la enseñanza obligatoria: prácticas escolares, actitudes y opiniones de padres, alumnos y profesores; resultados académicos de los estudiantes de diversos grupos culturales. Revista de Investigación Educativa, Jaén, v. 21, n. 2, p. 323-348, 2003. Disponible en: <http:// revistas.um.es/rie/article/viewFile/99241/94841>. Acceso en: 25 en. 2016.

BARRENA, María Hermenegilda. Actitudes del alumnado de educación secundaria obligatoria hacia la diversidad cultural. 2014. 334 p. Tesis (Doctorado en Estudios en Educación) - Facultad de Ciencias de la Educación, Universidad de Sevilla, Sevilla, 2014.

CALVO, Tomás. Actitudes ante la Inmigración y cambio de valores. Madrid: UCM, 2008.

DÍAZ, Sandra Milena. Los métodos mixtos de investigación: presupuestos generales y aporte a la evaluación educativa. Revista Portuguesa de Pedagogia, Coimbra, v. 48, n. 1, p. 7-23, 2014. D0I: 10.14195/16478614_481_1.

ETXEBARRIA, Felix; INTXAUSTI, Nahia. La percepción de tutores sobre la implicación educativa de familias inmigrantes en la comunidad autónoma del País Vasco. Revista Electrónica de Orientación Psicopedagógica, Madrid, v. 24, n. 3, p. 43-62, 2013. D0l: 10.5944/reop.vol.24.num.3.2013.11244.

ETXEBARRIA, Felix; INTXAUSTI, Nahia; JOARISTI, Luis. Factors Favouring the Educational Involvement of Immigrant Families with Children in Primary Education. Revista de Psicodidáctica, Madrid, v. 18, n. 19, p. 43-62, 2013. DOI: 10.1387/RevPsicodidact.5684.

ETXEBARRIA, Felix; KARRERA, Iñaki; MURUA, Hilario. Competencias interculturales del profesorado con alumnado inmigrantes en el País Vasco. Revista Electrónica Interuniversitaria de Formación del Profesorado, Murcia, v. 13, n. 4, p. 80-94, 2010. Disponible en: <http://www.aufop.com>. Acceso en: 1 my. 2016.

ETXEBERRIA, Felix et al. ¿Coinciden las expectativas escolares de la familia y del profesorado acerca del alumnado de origen inmigrante? Revista Electrónica de Investigación y Evaluación Educativa, Valencia, v. 20, n. 1, p. 1-21. 2014. DOl: 10.7203/ relieve.20.1.3804. 
FRANZÉ, Adela. Diversidad cultural en la escuela. Algunas contribuciones antropológicas. Revista de Educación, Madrid, v. 345, p. 111-132, 2008. Disponible en: <http://www.revistaeducacion.mec.es/ re345_05.html>. Acceso en: 10 abr. 2016.

GARRETA, Jordi. Escuela, familia de origen inmigrante y participación. Revista de Educación, Madrid, v. 345, p. 133-155, 2008. Disponible: <http://www.revistaeducacion.mec.es/re345_06.html>. Acceso en: 12 nov. 2015.

GÓMEZ, Inmaculada. Formación del profesorado para el tratamiento educativo de los conflictos sobre diversidad cultural y de género. 2015. 704 p. Tesis (Doctorado en Humanidades) - Facultad de Educación, Universidad de Madrid, Madrid, 2015.

INJUVE. Informe 2008. Madrid: Ministerio de Igualdad de España, 2008. Disponible en: <http://www.injuve. es/sites/default/files/9314-04.pdf>. Acceso en: 23 agt. 2018.

INTXAUSTI, Nahia; ETXEBARRIA, Felix; JOARISTI, Luis. ¿Coinciden las expectativas escolares de la familia y del profesorado acerca del alumnado de origen inmigrante? Revista Electrónica de Investigación y Evaluación Educativa, Valencia, v. 20, n. 1, p. 1-21, 2014. D0I: 10.7203/ relieve.20.1.3804.

LEIVA, Juan José. Interculturalidad, gestión de la convivencia y diversidad cultural en la escuela: un estudio de actitudes del profesorado. Revista Iberoamericana de Educación RIEOEI, v. 46, n. 2, p. 1-14, 2008. Disponible en: <http://rieoei.org/2297.htm>. Acceso en: 21 jun. 2016.

LEIVA, Juan José. La formación en educación intercultural del profesorado y la comunidad educativa. Revista Electrónica de Investigación y Docencia, p. 8-31, 0ct. 2012. Disponible en: <http://www.revistareid. net/monografico/n2/REIDM2art1.pdf>. Acceso en: 6 jul. 2016.

LEIVA, Juan José. Práctica de la interculturalidad desde la perspectiva docente: análisis y propuestas pedagógicas. Cultura y Educación, Barcelona, v. 22, n. 1, p. 67-84, 2010.

LEIVA, Juan José. Relaciones interpersonales en contextos de Educación Intercultural: un estudio cualitativo. Dedica, Coimbra, v. 4, p. 109-128. 2013. Disponible en: <https://dialnet.unirioja.es/servlet/ articulo?codigo=4252334>. Acceso en: 10 may. 2016.

LEIVA, Juan José; ESCARBAJAL, Andrés. La participación de las familias inmigrantes como fundamento pedagógico en la construcción de la interculturalidad en la escuela. Educatio XXI, Madrid, v. 29, n. 2, p. 389-416. 2011. Disponible en: <https://dialnet.unirioja.es/servlet/articulo?codigo=3719934>. Acceso en: 4 my. 2016.

MERINO, David; LEIVA, Juan José. El docente ante la realidad intercultural: análisis de sus actitudes para la comprensión de sus nuevas funciones. El Guiniguada, Las Palmas, v. 16, p. 80-95, 2007

MERINO, David; RUIZ, Cristóbal. Actitudes de los profesores hacia la educación intercultural. Aula Abierta, Oviedo, v. 86, p. 185-203, 2005. Disponible en: <https://dialnet.unirioja.es/servlet/ articulo?codigo=2328717>. Acceso en: 2 abr. 2016. 
MOLINER, Lidón; MOLINER, Odet. Percepciones del profesorado sobre la diversidad. Revista de Educación Inclusiva, Jaén, v. 3, n. 3, p. 23-33, 2010. Disponible en: <http://www.ujaen.es/revista/rei/linked/ documentos/documentos/11-2.pdf>. Acceso en: 10 abr. 2016.

RUIZ, Cristóbal; MERINO, David. La experiencia escolar de los hijos de inmigrantes marroquíes en los Centros de Educación Secundaria Obligatoria. Revista Electrónica Interuniversitaria de Formación del Profesorado, Salamanca, v. 12, n. 3, p. 87-97, 2009. Disponible en: <http://www.aufop.com/aufop/uploaded_files/ articulos/1254436191.pdf>. Acceso en: 20 abr. 2016.

SALAS,Ana etal. La educación intercultural percepciones y actitudes del profesorado. Revista lberoamericana de Educación de la 0EI, Madrid, v. 58, n. 1, p. 1-15, 2012. Disponible en: <http://rieoei.org/rie_contenedor. php?numero=4652\&titulo=La\%20educaci\%C3\%B3n\%20intercultural:\%20percepciones\%20y\%20 actitudes\%20del\%20profesorado>. Acceso en: 9 my. 2016.

SANTANA, Lidia E; FELICIANO, Luis; JIMÉNEZ, Ana B. Apoyo familiar percibido y proyecto de vida del alumnado inmigrante de educación secundaria. Revista de Educación, Madrid, v. 372, p. 35-62, 2016. D0I: 10.4438/1988-592X-RE-2015-372-314.

SANTOS, Miguel Ángel; LORENZO, Mar. La participación de las familias inmigrantes en la escuela. Un estudio centrado en la procedencia. Revista de Educación, v. 350, p. 277-300, 2009. Disponible en: <http://www.revistaeducacion.mec.es/re350/re350_12.pdf>. Acceso en: 5 my. 2016.

SANTOS, Miguel Ángel; LORENZO, Mar; PRIEGUE, Diana. Infancia de la inmigración y educación: la visión de las familias. Revista de Investigación Educativa, Murcia, v. 29, n. 1, p. 97-110, 2011. Disponible en: <http://revistas.um.es/rie/article/view/110351/126952>. Acceso en: 2 abr. 2016.

SETTON, Maria da Graça Jacintho. A estrutura da escola: uma perspectiva multidimensional. Educação e Pesquisa, São Paulo, v. 43, n. 3, p. 623-628, 2017. D0l: http://dx.doi.org/10.1590/s1517970220174303001.

Recibido en: 29.11.2017 Aprobado en: 12.01.2018

Antonio Rodríguez Fuentes es doctor en Ciencias de la Educación y profesor titular de la Universidad de Granada. Autor de libros en editoriales especializadas, así como de artículos en diversas revistas prestigiosas e internacionales. Ponente e investigador invitado en instituciones europeas, americanas y africanas.

Alejandro Daniel Fernández Fernández es doctor en Ciencias de la Educación, maestro funcionario y director de colegio CEIP Las Gaviotas, donde coordina varios proyectos educativos del Centro. Autor de algunos artículos en diferentes revistas y capítulos de libros en editoriales igualmente diversas, unas y otras de prestigio científico. 